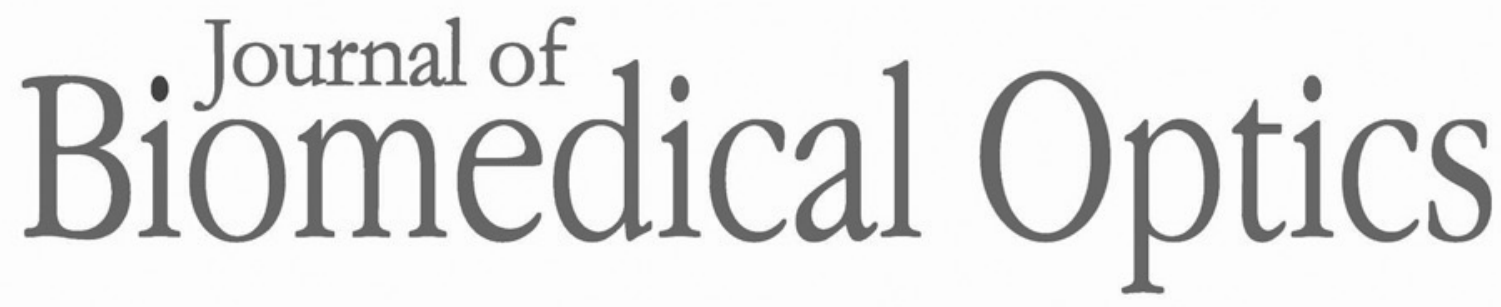

\title{
Optical coherence tomography-guided dynamic focusing for combined optical and mechanical scanning multimodal photoacoustic microscopy
}

\author{
Arash Dadkhah \\ Shuliang Jiao
}




\title{
Optical coherence tomography-guided dynamic focusing for combined optical and mechanical scanning multimodal photoacoustic microscopy
}

\author{
Arash Dadkhah and Shuliang Jiao* \\ Florida International University, Department of Biomedical Engineering, Miami, Florida, United States
}

\begin{abstract}
To achieve fast imaging and large field of view (FOV), we improved our multimodal imaging system, which integrated optical resolution photoacoustic microscopy, optical coherence tomography (OCT), and confocal fluorescence microscopy in one platform, by combining optical scanning with mechanical scanning. To ensure good focusing of the objective lens over all the imaged area, we employed OCT-guided dynamic focusing. Different from our previous point-by-point dynamic focusing, we employed an area-by-area focusing adjustment strategy, in which each fast optical scanning area has a fixed focusing depth. We have demonstrated the performance of the system by imaging biological samples ex vivo (plant leaf) and in vivo (mouse ear). The system has achieved uniform resolution in an FOV of $10 \mathrm{~mm} \times 10 \mathrm{~mm}$ with an imaging time of about $5 \mathrm{~min}$. (C) The Authors. Published by SPIE under a Creative Commons Attribution 4.0 Unported License. Distribution or reproduction of this work in whole or in part requires full attribution of the original publication, including its DOI. [DOI: 10.1117/1.JBO.24.12.121906]

Keywords: photoacoustic microscopy; optical coherence tomography; confocal fluorescence microscopy; dynamic focusing; multimodal imaging; optical coherence tomography guidance.
\end{abstract}

Paper 190175SSR received May 30, 2019; accepted for publication Jul. 29, 2019; published online Aug. 13, 2019.

\section{Introduction}

Photoacoustic microscopy (PAM) is an emerging biomedical optical imaging technology, which is capable of imaging the optical absorption contrast of biological tissues. PAM-based multimodal imaging technologies combine different imaging modalities to reveal complementary information of biological tissues for potential applications in both research and clinical diagnosis. PAM-based multimodal imaging has been achieved by combining PAM with other microscopic imaging modalities such as optical coherence tomography (OCT), optical Doppler tomography, confocal fluorescence microscopy (CFM), multiphoton microscopy, or second-harmonic generation microscopy. ${ }^{1-7}$ In these imaging techniques, the PAM and other imaging modalities may share the same pulsed light source. ${ }^{5,8-12}$

Field of view (FOV) and imaging speed are the two important parameters affecting the application of PAM-based multimodal imaging systems. Using optical scanning and an unfocused ultrasonic transducer, optical-scanning PAM can achieve real-time imaging with an imaging speed depending on the pulse repetition rate (PRR) of the excitation laser. However, in such a configuration, the FOV is limited by the field of sensitivity of the fixed unfocused ultrasonic transducer. One method to achieve faster imaging speed and large FOV is to combine optical scanning with mechanical scanning. ${ }^{13}$

Focusing of the objective lens is a key for achieving highquality microscopic imaging, especially for a large FOV. Dynamic focusing is necessary when the contour of the sample surface is uneven and has a range of depth variation in the FOV that exceeds the depth of focus of the objective lens. Without dynamic focusing, the uneven surface of a sample can lead to uneven depth of focus and results in images with nonuniform lateral resolution and signal intensity, which is more significant

*Address all correspondence to Shuliang Jiao, E-mail: shjiao@fiu.edu with increasing FOV. Dynamic focusing has been reported for PAM imaging using contour scan with PAM ${ }^{14}$ or ultrasound. ${ }^{15}$ We have achieved OCT-guided dynamic focusing for multimodal PAM imaging and demonstrated the capability by imaging biological samples in vivo. ${ }^{16}$ OCT-guided dynamic focusing intrinsically has better guiding accuracy and faster guiding speed due to the better spatial resolution and much faster imaging speed.

To achieve fast imaging and large FOV, we updated our multimodal imaging system, which integrated optical resolution PAM, OCT, and CFM in one platform, by combining optical scanning with mechanical scanning. Optical scanning performs a fast scan of the combined PAM excitation laser and the OCT probe light in the FOV of the needle ultrasonic transducer while mechanical scanning moves the imaging subject with a step size defined by the FOV of the transducer. The integrated OCT plays a key role not only for imaging but also for guiding the dynamic focusing to achieve uniform resolution in the combined large FOV. We took a different strategy for dynamic focusing with OCT guidance to match with the faster imaging speed.

\section{Methods}

\subsection{Imaging System}

A schematic of the experimental system is shown in Fig. 1, which is similar to that in our previous publication ${ }^{16}$ except that a pair of galvanometer scanning mirrors (shown in the dashed box in Fig. 1) was added in the optical systems to achieve fast scanning of the combined near-infrared (NIR) and visible light beams across the aperture of the objective lens.

In brief, the system combined PAM, OCT, and CFM for simultaneous multimodal imaging. PAM and CFM shared the same Q-switched frequency-doubled Nd:YAG laser (SPOT10-200-532, Elforlight Ltd., wavelength: $532 \mathrm{~nm}$, maximum pulse energy: $20 \mu \mathrm{J}$, pulse duration: $2 \mathrm{~ns}$, and maximum 


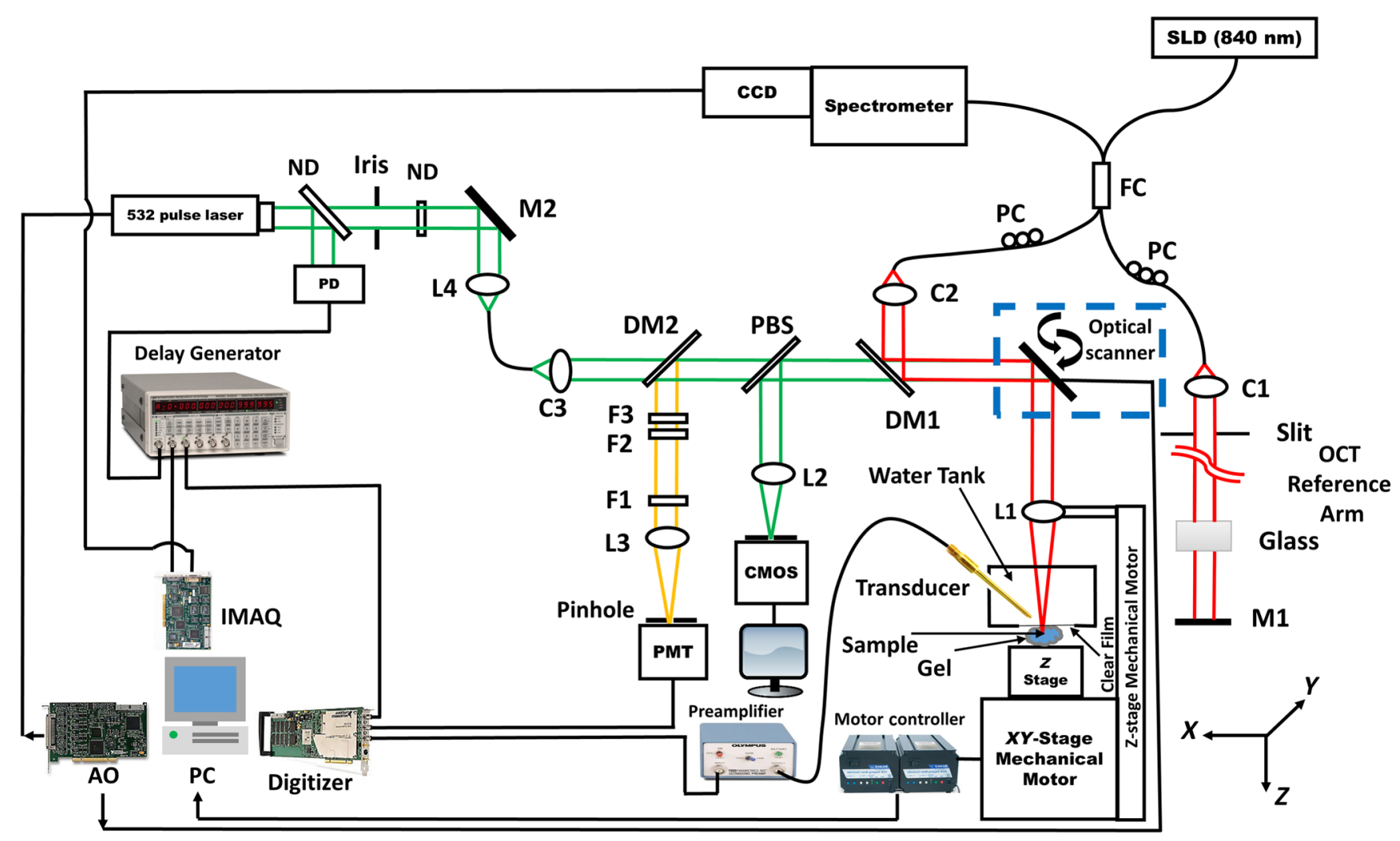

Fig. 1 Schematic of the integrated PAM/OCT/CFM experimental system. SLD: superluminescent diode; FC: $2 \times 2$ fiber coupler; PC: polarization controller; L1, L2, L3, L4: lenses; M1, M2: mirrors; DM1, DM2: dichroic mirrors; C1, C2, C3: collimators; AO: analog output; ND: neutral density; F1, F2, F3: optical filters; PBS: pellicle beam splitter; and PD: photodiode.

PRR: $30 \mathrm{kHz}$ ), while the optical fiber-based OCT used a broadband superluminescent diode-based light source (T-840 Broadlighter, Superlum Diodes Ltd., Ireland). The output pulsed laser was combined with the light beam in the sample arm of the OCT by a dichroic mirror (DM1). The combined light was scanned by the $X-Y$ galvanometer mirrors and focused onto the sample by an objective lens (LMH-5X-532, EFL $=40 \mathrm{~mm}$, $\mathrm{NA}=0.13$, Thorlabs). The OCT signal was detected in the spectral domain by a homemade spectrometer. The fluorescence signal was detected by a PMT (PMM02, Thorlabs) with a confocal arrangement through a set of filters and dichroic mirrors, which limited the detection spectrum to 550 to $750 \mathrm{~nm}$. The generated photoacoustic signal from the sample was detected by an unfocused needle ultrasonic transducer, which was immersed in a water tank filled with distilled water. The transducer has a center frequency of $30 \mathrm{MHz}$ with an active element diameter of $3 \mathrm{~mm}$. The performances of all the subimaging systems are similar to that in our previous publication. ${ }^{16}$

We combined optical scanning with mechanical scanning to increase the imaging speed and the FOV. The scanning directions of the galvanometer mirrors are aligned with the mechanical scan. Mechanical scanning was accomplished using a stepmotor-driven platform to move the sample together with the water tank and the ultrasonic transducer in the lateral directions $(X$ and $Y$ ). The objective lens was mounted on a $Z$-scan platform for fine-tuning the focus with guidance of the OCT.

All the imaging systems were synchronized using the sampling clock of an analog output board (PCI-6731, National Instruments), the outputs of which controlled the $X-Y$ galvanometers. At each scanning position of the galvanometers, the sampling clock triggered the pulsed laser, which was partially reflected by a glass plate and detected by a photodiode (PD). The output of the PD triggered a multichannel digital delay generator (DG645, Stanford Research Systems). One output channel of the delay generator was used to trigger the image acquisition board (PCI-1428, National Instruments) for acquiring the interference spectrum of OCT and another channel was used to trigger the digitizer for acquiring the PAM and CFM signals.

\subsection{Scanning Scheme and Optical Coherence Tomography-Guided Dynamic Focusing}

To achieve large FOV and maximize imaging speed with minimal loss of signal-to-noise ratio (SNR), the laser and NIR beams are first scanned within the FOV of the ultrasonic transducer $(\sim 2 \mathrm{~mm} \times 2 \mathrm{~mm})$ by the galvo-mirrors. The sample is then moved to the adjacent imaging area by the mechanical scanning platform. This procedure repeats until the entire region of interest (ROI) of the sample is imaged. At each interval between the mechanical and the optical scanning, the objective lens is adjusted for dynamic focusing. Once the entire ROI of the sample is scanned, all the small images acquired with fast optical scanning are stitched together to form an image of large FOV.

For dynamic focusing, we first established the depth relationship between the PAM and the OCT by imaging a black tape. The maximum detected photoacoustic signal is achieved when 
the objective lens is in focus while the OCT image provides the depth of the surface. The relationship of the optical focus of the objective lens with depth can thus be established. In our previous mechanical scanning multimodal imaging system, OCT-guided dynamic focusing was applied point by point, i.e., the focus of the objective lens was adjusted for each scanning position. This point-by-point dynamic focusing method is suitable for the relatively slow mechanical scanning mechanism. For the current fast optical scanning, the adjusting speed of the objective lens cannot catch up with the scanning speed. Thus, we adopted an aerial dynamic focusing strategy, i.e., the focus of the objective lens is adjusted area by area.
To achieve dynamic focusing, we need to establish a reference focal plane for the objective lens. For biological imaging, we manually adjusted the sample to reach the maximum photoacoustic signal at a reference point, which was selected with the help of the real-time video image and PAM B-scans. The depth location of this reference point is extracted by OCT as a reference for the subsequent scans. In each fast scanning area, the sample was first imaged with OCT. The depth of the sample surface at each OCT A-line was extracted and the depth position ( $Z$ coordinates) was averaged. The difference between this averaged $Z$ position and that of the reference point gives the adjustment range of the objective lens. (a)
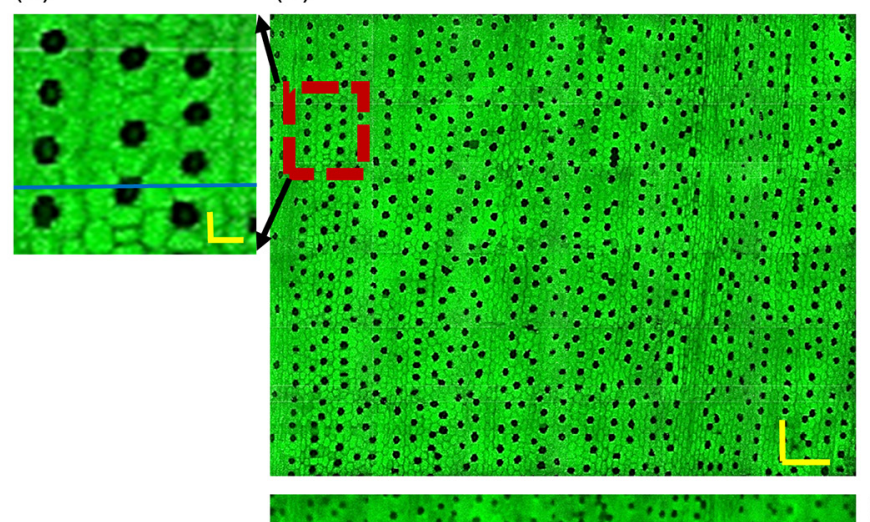

(b)

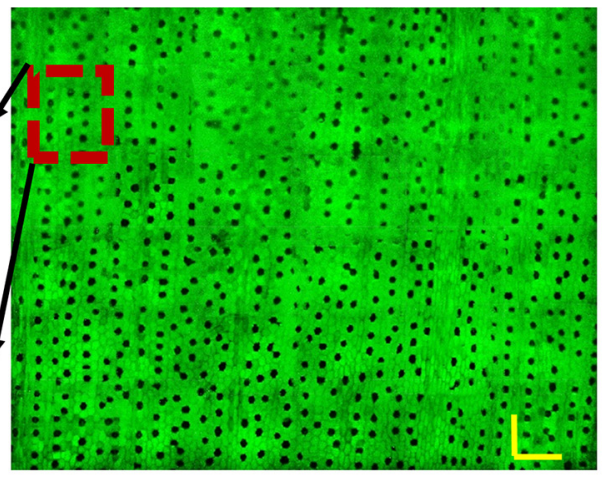

(f)
Scale bar for large figures $(b, c, f$ and $g): 1 \mathrm{~mm}$ Scale bar for small figures $(a, d, e$ and $h): 200 \mu m$ (c)
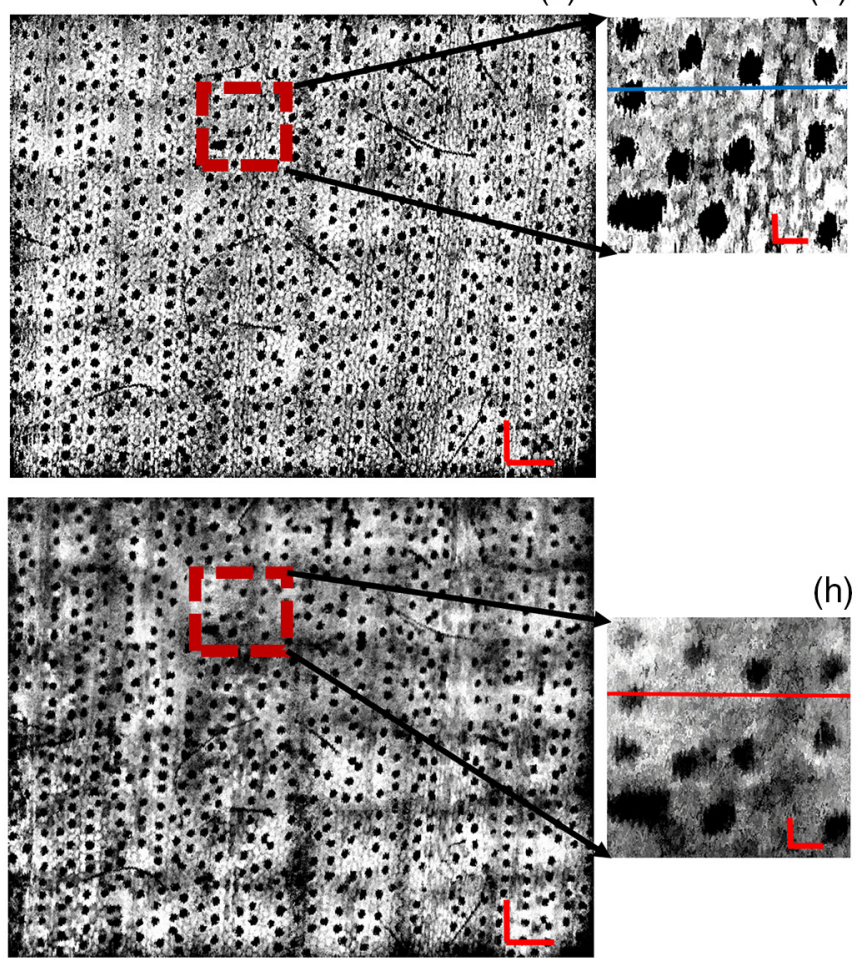

(g)

(h)

d)

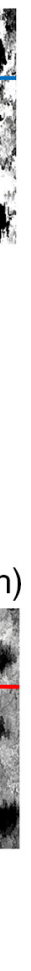

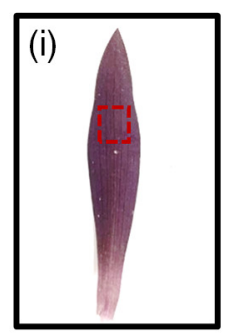
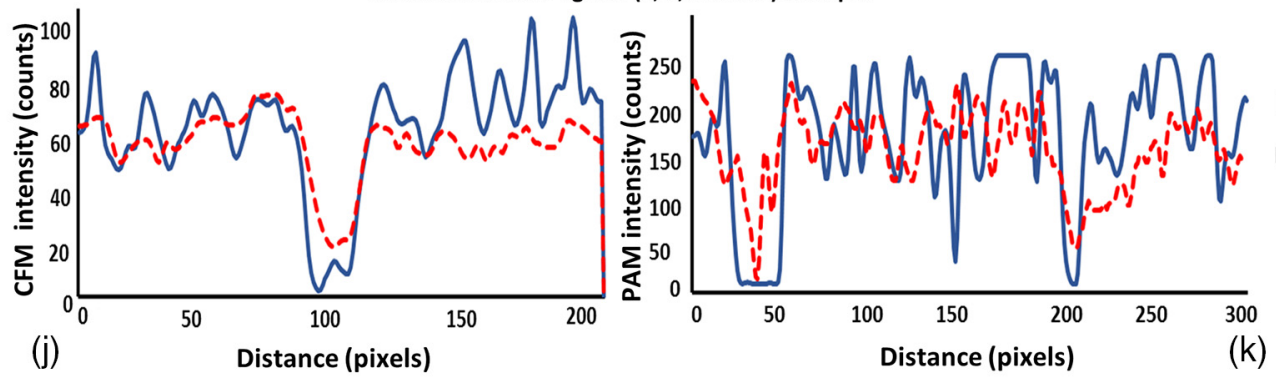

\section{.}

Fig. 2 Simultaneously acquired PAM and CFM images of a purple queen plant leaf to test the OCTguided dynamic focusing. (a) Enlarged CFM image with dynamic focusing marked in panel (b) by a dashed box; (b) CFM image with dynamic focusing; (c) maximum amplitude projection of the PAM image with dynamic focusing; (d) enlarged PAM image with dynamic focusing marked in panel (c) by a dashed box; (e) enlarged CFM image without dynamic focusing marked in panel (f) by a dashed box; (f) CFM image without dynamic focusing; (g) PAM image without dynamic focusing; (h) enlarged PAM image without dynamic focusing marked in panel (g) by a dashed box; (i) a photo of the imaged sample, where the selected imaging area is marked by a dashed box; (j) fluorescence signal intensity along the lines at the location marked in panels (a) and (e) with (blue) and without (red) dynamic focusing, respectively; (k) photoacoustic signal intensity along the lines at the location marked in panels (d) and (h) with (blue) and without (red) dynamic focusing, respectively; bar: $1 \mathrm{~mm}$ and $200 \mu \mathrm{m}$ for large and small figures, respectively. 
We have proved that the mean of the $Z$ coordinates of the scanned sample surface equals the minimum of the mean squared error. The depth positions of the sample surface at each scanning point and the optimal point for focusing adjustment at a fast scanning area are expressed as $Z_{\mathrm{s}}$ and $Z_{\mathrm{o}}$, respectively. The sum of the squared error can be expressed as

$\Delta z^{2}=\sum_{m=1}^{M}\left[z_{\mathrm{s}}(m)-z_{\mathrm{o}}\right]^{2}$,

where $m$ is the sequential number of a scanning point and $M$ is the total number of the scanning point in the fast scanning area. When the optimal depth adjustment is selected for $Z_{0}$, we should have $\frac{\mathrm{d} \Delta Z^{2}}{\mathrm{~d} Z_{0}}=0$. We thus have

$z_{\mathrm{o}}=\frac{\sum_{m=1}^{M} Z_{\mathrm{s}}(m)}{M}$

\section{Results}

The scanning scheme and the performance of dynamic focusing were validated by imaging various biological samples. All experimental animal procedures were conducted in compliance with the guidelines of the Florida International University's Institutional Animal Care and Use Committee.

We first imaged a leaf of purple queen plant as shown in Fig. 2(i). The leaf was selected because of its availability of providing contrasts for all the imaging modalities. It also has abundant structures to provide easy visual judgment about the performance of dynamic focusing. The freshly collected leaf was taped on an aluminum plate and placed under the window of the water tank. Ultrasonic coupling gel was applied between the leaf and the polystyrene film of the window to ensure good ultrasound coupling. The sample was manually adjusted to the focal plane of the objective lens while monitoring the OCT and PAM signals and the reference point was defined for dynamic focusing accordingly. Each fast imaging area consisted of $512 \times$ 128 A-lines covering an area of $2 \mathrm{~mm} \times 2 \mathrm{~mm}$ contributing to the final large area of $12 \mathrm{~mm} \times 12 \mathrm{~mm}$. The entire image acquisition took about 8 min.

Figure 2 shows the CFM and PAM imaging results of the leaf with [Figs. 2(b) and 2(c)] and without [Figs. 2(f) and 2(g)] dynamic focusing. The CFM and PAM images with large FOV of $12 \mathrm{~mm} \times 12 \mathrm{~mm}$ consist of 36 small images acquired with fast optical scanning. We montaged the small fast scanning images using ImageJ. According to the literature, the signals in PAM and CFM were generated by anthocyanin and chlorophylls in the leaf structure, respectively. From the enlarged images shown in Figs. 2(a), 2(d), 2(e), and 2(h), we can clearly see the improved sharpness of the CFM and PAM images with dynamic focusing. With dynamic focusing, the nuclei and cell walls are clearly seen in the images. In addition, the signal intensity of the PAM and CFM images is significantly improved across the large imaging area. For a direct comparison to show the improvement, we plotted the signal intensities of both CFM and PAM [Figs. 2(j) and 2(k)] along the lines marked in Figs. 2(a), 2(d), 2(e), and 2(h). We calculated the estimated contrast by subtracting the averaged signal intensity inside the cells that appeared dark from the averaged signal intensities outside the cells that appeared bright for both CFM and PAM. The estimated contrast improvement is $41 \%$ and $38 \%$ for CFM and PAM, respectively.
We imaged the ear of a Swiss Webster mouse in vivo to demonstrate the full potential of the multimodal imaging system with dynamic focusing for combined large FOV and fast imaging speed. We first anesthetized the animal by intraperitoneal injection of a cocktail containing ketamine $(54 \mathrm{mg} / \mathrm{kg}$ body weight) and xylazine ( $6 \mathrm{mg} / \mathrm{kg}$ body weight). Then, we gently removed the hair from the ear using a commercial hair removal gel and applied a drop of acoustic coupling gel on the mouse ear. The mouse was restrained in a homemade animal mount, which was fixed on the manually adjustable $Z$ axis stage (Fig. 1). The mouse ear was in contact with the polystyrene film window of the water tank through ultrasonic coupling gel. The sample was moved to the focal plane with the guidance of the real-time OCT. The ROI was selected with the help of the real-time microscopic imaging. Following the same procedure for reference point selection and dynamic focusing, the sample was imaged with a total FOV of $10 \mathrm{~mm} \times 10 \mathrm{~mm}$, which took about 5 min for the whole image acquisition.

Figure 3 shows the simultaneously acquired PAM, CFM, and OCT images of a mouse ear with OCT-guided dynamic focusing. The images of different imaging modalities revealed complementary features of the mouse ear as a result of the different contrast mechanisms provided. The PAM image in Fig. 3(a) clearly reveals the blood vessels, the imaging contrast of which is provided by the optical absorption of hemoglobin in the red blood cells. Figure 3(b) displays the fluorescence image, which revealed the sebaceous glands distributed in the ear skin. The possible sources of autofluorescence in the sebaceous glands
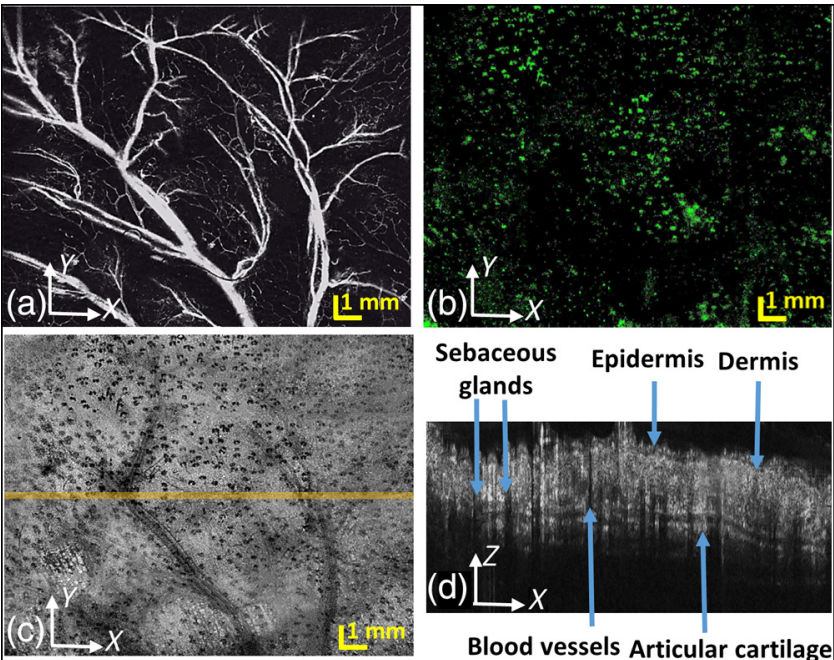

Blood vessels Articular cartilage
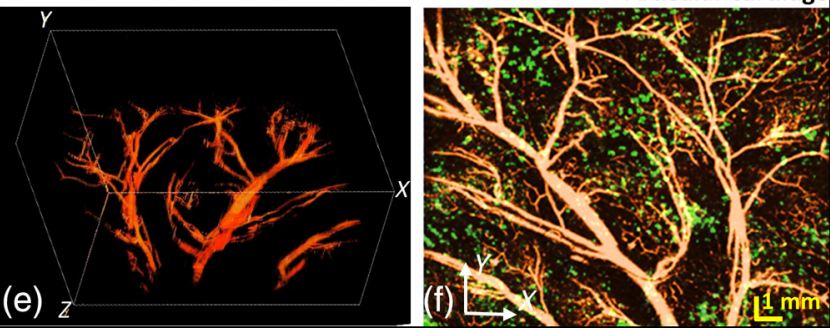

Fig. 3 Simultaneously acquired PAM, CFM, and OCT images of a mouse ear with dynamic focusing. (a) Maximum amplitude projection of the PAM image; (b) CFM image; (c) OCT projection images generated from the acquired 3-D OCT datasets; (d) OCT B-scan at the location marked in panel (c) by a solid line; (e) PAM 3-D image reconstruction; and (f) fused PAM projection and CFM images; SG, sebaceous glands; bar: $1 \mathrm{~mm}$. 
include porphyrins, keratin, and tryptophan molecules. ${ }^{17,18}$ Figure 3(c) shows the en face view of the three-dimensional (3-D) OCT dataset displaying in the $X-Y$ plane. Due to the shadowing effect as shown in the OCT B-scan image [Fig. 3(d)], the sebaceous glands appear as black spots in the en face OCT display. The OCT cross-sectional imaging in Fig. 3(d) shows the depth resolved tissue structures. Figure 3(e) shows a 3-D reconstruction of the PAM image. Figure 3(f) illustrates the fused image combining simultaneously acquired CFM and PAM images. Due to dynamic focusing, the image resolution is uniform across all the large FOV, which is evidenced in Fig. 3(a), where capillaries can be recognized in most of the imaged area.

\section{Discussions}

By combining optical and mechanical scanning, we have significantly improved the imaging speed for large FOV imaging. The imaging speed is mainly limited by the PRR of the PAM excitation laser and the speed of the mechanical scan. Thus, to further improve the imaging speed, a higher PRR laser and a faster scanning motor are needed. Currently, the PRR of a commercially available Q-switched laser can reach $>100 \mathrm{kHz}$. Such a high PRR laser will greatly improve the overall imaging speed. In the current imaging system, the OCT imaging speed is matched with that of the PAM and CFM. The OCT imaging speed can be easily increased to $100 \mathrm{kHz}$ with a fast CMOS camera used in the spectrometer.

OCT-guided dynamic focusing has enabled uniform focusing of the objective lens across the large FOV. However, there is a possible tradeoff between effective focusing and the fast optical scanning FOV. In case the contour of the sample surface in the optical scanning FOV has a depth-variation range exceeding the depth of focus of the objective lens, we will need to reduce the area of optical scanning to ensure all points in the optical scanning FOV will be in focus. Thus, to determine the optical scanning FOV, an evaluation of the surface condition with OCT may be necessary before acquisition of the multimodal images.

The ultrasonic transducer also plays a key role in determining the PAM imaging quality, the optical scanning range, and thus the overall imaging speed. In Fig. 3(a), we can see that the image has a resolution down to the capillary level. However, not all the capillaries were resolved in the FOV, which is hypothesized to be the result of limited sensitivity of the transducer. The field of sensitivity of transducer limits the FOV of the optical scan. In Figs. 2(c) and 2(g), it appears that the edges of each fast scanning area have relatively lower intensity, which is caused by the relatively low sensitivity of the ultrasound transducer at the edge of its FOV. One possible solution would be reducing the step size of the mechanical motor creating larger overlap between each fast scanning so that it could compensate the dark edges and make the entire large image more uniform. When a transducer with larger FOV is used, the solution should be more feasible. Another solution is to use image processing to compensate for the sensitivity distribution.

The accuracy of surface contour extraction is determined not only by the axial resolution of the OCT but also the surface condition of the sample. As long as the sample surface can provide a clear boundary for OCT imaging, there would be no problem for extracting the depth of the surface at each OCT A-line. Since dynamic focusing is following the contour of the sample surface, we also assume that the targeted tissue structure for imaging, such as the vasculature network, is parallel to the sample surface or the depth variation in reference to the sample surface is less than the depth of focus of the objective lens. This assumption works for the mouse ear sample because we have good focusing across the whole large FOV.

Since only the focus of the objective lens is adjusted and the sample remains stationary in $Z$ direction during imaging, the 3-D relationship among all the imaging points remains unchanged by dynamic focusing. Thus, 3-D reconstruction is not affected by the dynamic focusing process and there is no need for a large-scale alignment between the small images in the montage process. To reconstruct the final large 3-D PAM image, we first transferred every small 3-D data volume $(512 \times 128 \times 1000)$ into a single large 3 -D array $(2560 \times$ $640 \times 1000)$ for further image processing. After creating the large 3-D array, we extracted PAM vertical $(x-z)$ slices from the time-resolved photoacoustic signal at each depth, similar to the OCT B-scan. Then, each sliced image was enhanced to increase the SNR and reduce the possible artifacts rendering the final volume image by $Z$-stacking of all $(x-z)$ slices. However, the very small capillaries shown in Fig. 3(a) still did not appear in Fig. 3(e) because the low SNR created a noisy environment for 3-D reconstruction. Using an ultrasound transducer with higher SNR and also applying more efficient image processing methods will potentially improve the final 3-D PAM image in our future studies.

\section{Conclusion}

In conclusion, a combined optical and mechanical scanning multimodal PAM with OCT-guided dynamic focusing has been developed. The imaging system integrates PAM, OCT, and CFM images with complementary contrasts for multimodal imaging of biological tissues. By combining optical and mechanical scanning, the proposed imaging system has been able to achieve large FOV and high imaging speed. In addition, the OCT-guided dynamic focusing has been shown to be able to improve SNR across the entire imaged area of a sample with uneven surface. Ex vivo and in vivo imaging studies were performed and validated the capability of the proposed imaging system.

\section{Disclosures}

The authors have no relevant financial interests in this article and no potential conflicts of interest to disclose.

\section{Acknowledgments}

This work was supported in part by the National Institutes of Health Grant No. R01EY026643.

\section{References}

1. S. Jiao et al., "Simultaneous multimodal imaging with integrated photoacoustic microscopy and optical coherence tomography," Opt. Lett. 34(19), 2961-2963 (2009).

2. S. Jiao et al., "Photoacoustic ophthalmoscopy for in vivo retinal imaging," Opt. Express 18(4), 3967-3972 (2010).

3. L. Li et al., "Fast subcellular optical coherence photoacoustic microscopy for pigment cell imaging," Opt. Lett. 40(19), 4448-4451 (2015).

4. H. Fatakdawala et al., "Multimodal in vivo imaging of oral cancer using fluorescence lifetime, photoacoustic and ultrasound techniques," Biomed. Opt. Express 4(9), 1724-1741 (2013).

5. Y. Wang et al., "Integrated photoacoustic and fluorescence confocal microscopy," IEEE Trans. Biomed. Eng. 57(10), 2576-2578 (2010).

6. W. Song et al., "Fully integrated reflection-mode photoacoustic, two-photon, and second harmonic generation microscopy in vivo," Sci. Rep. 6, 32240 (2016). 
7. X. Liu et al., "Optical coherence photoacoustic microscopy for in vivo multimodal retinal imaging," Opt. Lett. 40(7), 1370-1373 (2015).

8. X. Liu et al., "Optical coherence photoacoustic microscopy (OC-PAM) with an intensity-modulated continuous-wave broadband light source," J. Opt. 18(6), 064001 (2016)

9. C. Lee et al., "Combined photoacoustic and optical coherence tomography using a single near-infrared supercontinuum laser source," Appl. Opt. 52(9), 1824-1828 (2013).

10. X. Zhang et al., "Simultaneous dual molecular contrasts provided by the absorbed photons in photoacoustic microscopy," Opt. Lett. 35(23), 4018-4020 (2010).

11. X. Zhang et al., "Simultaneous in vivo imaging of melanin and lipofuscin in the retina with photoacoustic ophthalmoscopy and autofluorescence imaging," J. Biomed. Opt. 16(8), 080504 (2011).

12. X. Zhang, S. Jiao, and H. Zhang, "Optical coherence photoacoustic microscopy: accomplishing optical coherence tomography and photoacoustic microscopy with a single light source," J. Biomed. Opt. 17(3), 030502 (2012).

13. B. Rao et al., "Hybrid-scanning optical-resolution photoacoustic microscopy for in vivo vasculature imaging," Opt. Lett. 35(10), 1521-1523 (2010).

14. C. Yeh et al., "Microvascular quantification based on contour-scanning photoacoustic microscopy," J. Biomed. Opt. 19(9), 096011 (2014).

15. B. Ning et al., "Ultrasound-aided multi-parametric photoacoustic microscopy of the mouse brain," Sci. Rep. 5, 18775 (2015).
16. A. Dadkhah et al., "Integrated multimodal photoacoustic microscopy with OCT-guided dynamic focusing," Biomed. Opt. Express 10(1), $137-150$ (2019)

17. W. H. Jang et al., "In vivo characterization of early-stage radiation skin injury in a mouse model by two-photon microscopy," Sci. Rep. 6, 19216 (2016).

18. C. Li et al., "Multiphoton microscopy of live tissues with ultraviolet autofluorescence," IEEE J. Sel. Top. Quantum Electron. 16(3), 516523 (2010).

Shuliang Jiao received his PhD from the Department of Biomedical Engineering of Texas A\&M University in 2003. Currently, he is a professor in the Department of Biomedical Engineering, Florida International University. His research interest is mainly focused on the development of innovative technologies for imaging and treatment of blinding eye diseases.

Arash Dadkhah is a fourth-year PhD candidate in Dr. Shuliang Jiao's lab in the Department of Biomedical Engineering at Florida International University. He received his master's degree in bioengineering with emphasis on biomechanics from Amirkabir University of Technology in Tehran, Iran. His PhD research is to combine different optical imaging modalities, including photoacoustic microscopy, optical coherence tomography, optical coherence angiography, optical Doppler tomography and fluorescence microscopy for simultaneous multimodal imaging of biological samples. 